\title{
Comparison of Knee Cruciate Ligaments Models Using Kinematics from a Living Subject during Chair Rising-Sitting
}

\author{
Rita Stagni, Silvia Fantozzi, Mario Davinelli, and Maurizio Lannocca \\ Department of Electronics, Computer Science and Systems, University of Bologna, \\ Viale Risorgimento 2, 40136 Bologna, Italy \\ \{rstagni, sfantozzi, maavinelli\}@deis.unibo.it \\ mlannocca@libero.it \\ http: //www-bio.deis.unibo.it/
}

\begin{abstract}
The knee joint is a key structure of the human locomotor system. Any lesion or pathology compromising its mobility and stability alters its function. As direct measurements of the contribution of each anatomical structure to the joint function are not viable, modelling techniques must be applied. The present study is aimed at comparing cruciate ligaments models of different complexity using accurate parameters from RMN and 3D-fluoroscopy of a single selected subject during chair rising-sitting motor task.. The complexity of the model was not relevant for the calculation of the strain range of the cruciate ligaments fibres. On the other hand, three-dimensionality and anatomical twist of the modelled fibres resulted to be fundamental for the geometrical strain distribution over the ligament section.
\end{abstract}

\section{Introduction}

The knee plays a fundamental role in determining the human locomotor ability. Any alteration of its anatomical structures can compromise its function. The development of effective methods for surgical reconstruction and rehabilitation is of great clinical interest, regarding both joint replacement and surgical reconstruction of the main anatomical structures. This interest is demonstrated by the 259000 total knee replacements, 25000 ligaments reconstructions and 15000 other repairs of the knee performed in the USA in 1997 as reported by the American Association of Orthopaedic Surgeons (AAOS). For the development of these procedures, an accurate knowledge of the mobility and stability of the whole articular structure, as well as of its different anatomical sub-units, is necessary. The need for this deeper knowledge led to a bulk of in-vitro and in-vivo studies, which allowed to clarify several aspects of the physiological behaviour of this complex joint. In-vitro testing allows to directly observe and measure different aspects of joint mechanics, but not in physiological conditions. During its normal function, the knee lets the shank move with respect to the thigh, maintaining the stability of the structure under articular load and torque. These are the result of several contributions: the inter-segmental contact load, ligament tensioning, loads applied by the muscles, the inertia of body segments. All these contributions are strongly dependent on the analysed motor task, as well as on 
the physical characteristics of the subject. Thus, if we want to quantify the contribution of each anatomical structure in determining the physiological function of the knee, modelling is the only possible solution, as direct measurements cannot be performed.

The problem of knee modelling has been approached at different levels of complexity. Two-dimensional models were designed in order to investigate the role of the cruciate ligaments in simple conditions, such as isometric quadriceps contraction $[1,2]$. Three-dimensional models, including articular surfaces and ligaments, were also proposed. Even these more complex models were applied in conditions far away from those of the physiological knee $[3,4,5,6]$. The natural evolution of this approach is inserting the model into a context which allows to evaluate the boundary conditions of the knee-structure during the performance of a simple task of daily living [7]. Even if the model is designed properly for the application devised, its potentials can be nullified by the effect of errors within the definition of subject parameters and during the acquisition of experimental inputs. In previous modelling attempts, these errors were due to discrepancies in the origin of parameters and inputs, which were often obtained from different and non-homogeneous subjects.

In order to avoid this possible source of error, in this paper, different cruciate ligament models were compared using parameters from a single selected subject analysed as accurately as possible. The specific geometry of articular surfaces and ligaments insertions were reconstructed using the three-dimensional reconstruction of segmented bone and soft tissues, obtained from Nuclear Magnetic Resonance (NMR). The specific accurate kinematics was obtained from cine-fluoroscopic images of a chair rising-sitting motor task. Cruciate ligaments models of different complexity: from the simple bi-dimensional untwisted one to the more realistic three-dimensional twisted with circular insertion were compared. The aim was to select the best compromise between accurate anatomical description and model simplicity for the investigation of knee biomechanics.

\section{Material and Methods}

Overview. A subject-specific model of the right knee of a young male living subject (height $168 \mathrm{~cm}$, weight $62 \mathrm{~kg}$, and age 30 years) was developed from a high resolution NMR data set. Three-dimensional outer surfaces of the biological structures of interest were generated.

The subject performed chair rising-sitting with the knee under analysis inside the fluoroscopic field of view. The accurate $3 \mathrm{D}$ pose of the bones was reconstructed by means of single-plane lateral 2D fluoroscopic projections and relevant models previously obtained.

The cruciate ligaments fibres were modelled with six geometrical equivalents and relative fibres strain compared: 2D, 3D with rectangular insertions, 3D with circular insertion, each twisted and untwisted.

The NMR data set. A data set of high resolution NMR images was collected with a 1.5T Gemsow scanner (GE Medical Systems, Milwaukee, Wisconsin). Details of the scanning parameters are shown in Table 1. 
Table 1. The NMR scanning procedure parameters

\begin{tabular}{ll}
\hline Scanning sequence & Spin Echo $(\mathrm{T} 1$ weighted) \\
Number of slices & 54 \\
Pixel spacing & $0.037 \mathrm{x} 0.037(\mathrm{~cm} \cdot \mathrm{cm})$ \\
Scanned region length (across the knee) & $15.9(\mathrm{~cm})$ \\
Slice thickness & $2.5(\mathrm{~mm})$ \\
Slice spacing & $3(\mathrm{~mm})$ \\
\hline
\end{tabular}

The segmentation procedure. A 3D tiled surface geometrical representation was generated using the software Amira (Indeed - Visual Concepts GmbH, Berlin, Germany), for the distal femur, the proximal tibia, and the insertion areas of the anterior (ACL) and posterior cruciate ligaments (PCL).

A segmentation of the NMR data set was performed with an entirely manual 2D segmentation technique. For each slice, the outer contour of the structures of interest was detected and outlined, as shown in Fig. 1. The resulting stacks of contours were interpolated to generate polygonal surfaces which represent the outer boundary of the objects to be modelled. The model used for the kinematic analysis is shown in Fig. 2.

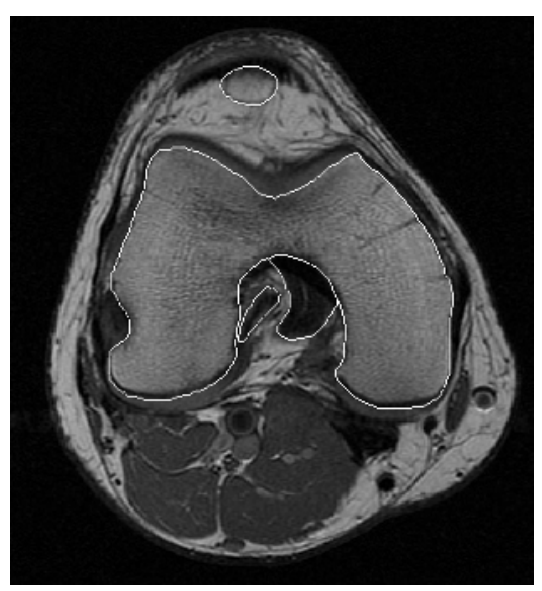

Fig. 1. Outlined contours of femur and ligaments in a slice of the NMR data set

Kinematics. Series of lateral images were acquired at the frequency of 6 samples per second with a standard fluoroscope (SBS 1600, Philips Medical System Nederland B.V.). Images of a 3D cage of Plexiglas with 18 tantalum balls in known positions and of a rectangular grid of tin-leaded alloy balls $5 \mathrm{~mm}$ apart were collected in order to calculate respectively the position of the camera focus and the parameters necessary for image distortion correction. This was obtained using a global spatial warping technique[8]. An established technique for 3D kinematics analysis of a known object from a single view was implemented [9] (Fig. 3). Bone poses in space were obtained from each fluoroscopic image by an iterative procedure using a technique based on tangent condition between projection lines and model surface. 
Previous validation work on prosthesis components [9] showed that relative pose can be estimated with an accuracy better than 1.5 degrees and $1.5 \mathrm{~mm}$.

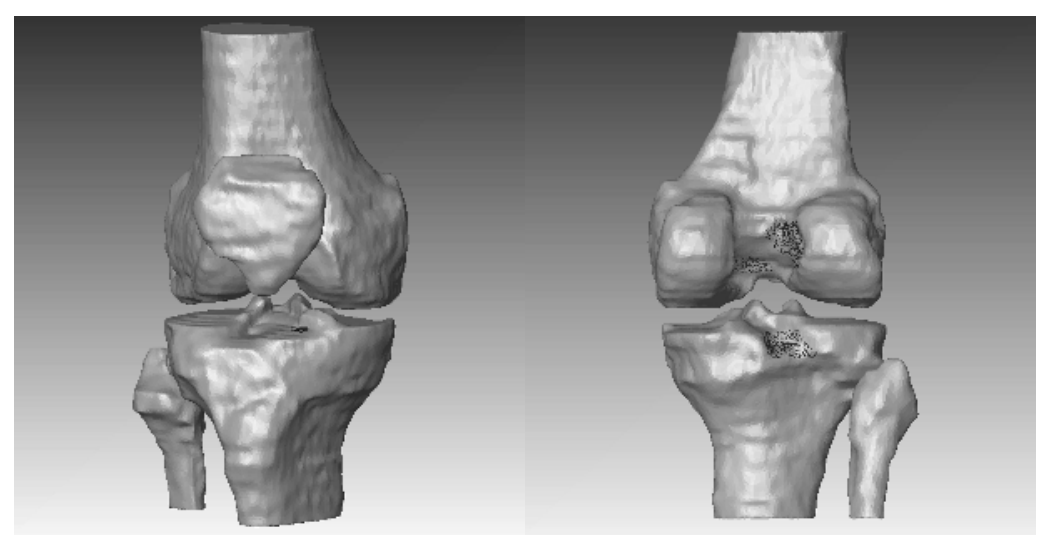

Fig. 2. Anterior and posterior view of the complete knee model. The areas of insertion of ligaments are the dotted regions on the femur and the tibia

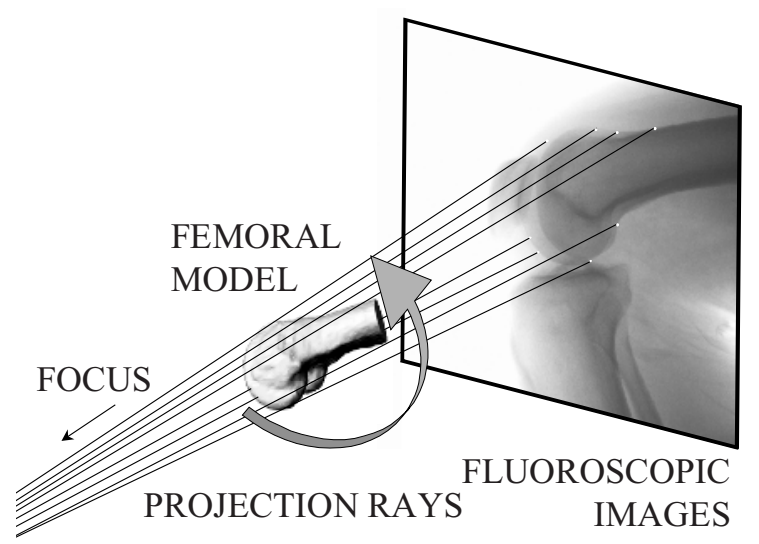

Fig. 3. Sketch of the model for fluoroscopic image generation process

Cruciate ligament geometrical models. The geometrical models of the cruciate ligaments differ for dimension, shape of insertion and twist:

1. Bi-dimensional - untwisted. The insertions were modelled as follows: a) the line that minimizes in a least squares sense the points of the insertion area was calculated, b) insertion segment was identified on this line between the anterior and posterior limits of the insertion surface, and c) 25 uniformly distributed points were identified on this segment. Thus, 25 fibres were modelled for both ACL and PCL. In both ligaments the fibres connected these points of the insertions from the most 
posterior to the most anterior on both femur and tibia, i.e. the most posterior point of femur insertion was connected to the most posterior point of the tibia insertion.

2. Bi-dimensional - twisted. The insertions and the fibre were modelled as in model 1 except for the fact that the points from the most posterior to the most anterior of the femur were connected to those from the most anterior to the most posterior of the tibia.

3. Three-dimensional - rectangular insertions - untwisted. The insertions were modelled as follows: a) in the plane approximating the insertion points in a least square sense a rectangle including $80 \%$ of these points was estimated, and b) a $5 \times 5$ uniform grid of points was identified on the rectangle. In both ACL and PCL the 25 fibres connected points of the insertions with no twisting.

4. Three-dimensional - rectangular insertions - twisted. The insertions and the fibre were modelled as in model 3 except for the fact that in both ligaments a twist angle of $90^{\circ}$ was introduced.

5. Three-dimensional - circular insertions - untwisted. The insertions were modelled as follows: a) in the plane approximating the insertion points in a least square sense a circle including $80 \%$ of these points was estimated, and b) a 25 uniformly distributed points were identified on the circle. In both ACL and PCL the 25 fibres connected points of the insertions with no twisting.

6. Three-dimensional - circular insertions - twisted. The insertions and the fibre were modelled as in model 5 except for the fact that in both ligaments a twist angle of $90^{\circ}$ was introduced.

For each model, for each single fibre, the strain, $\mathcal{E}$, was calculated as follows:

$$
\varepsilon(t)=\frac{L(t)-L_{0}}{L_{0}}
$$

were $L$ is the length of the fibre at time sample $t$, and $L_{0}$ is the maximum length the fibre reached during the motor task.

\section{Results}

The modelled PCL always showed a larger elongation, with an average strain of about $33 \%$ versus $19 \%$ of the ACL. The strain calculated for the fibre approximately connecting the mean point of the insertions of the ACL and PCL was equivalent for all ligament models. The range of the strains calculated for the ACL $[-16 \% ;-20 \%]$ and for the PCL fibres $[-24 \% ;-38 \%]$ was similar for the different models. The geometrical distribution of the strain over the ligament section resulted model-dependent. 


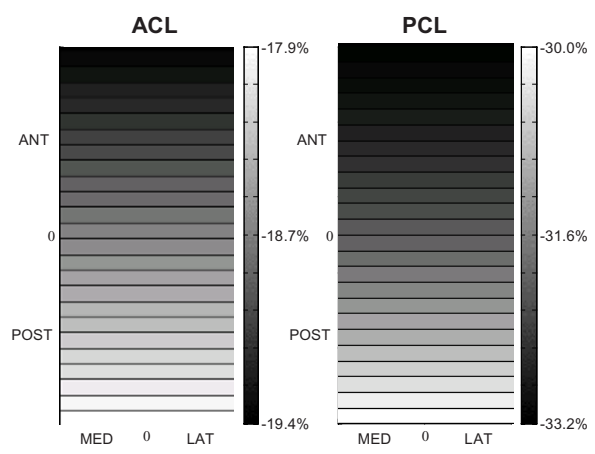

a

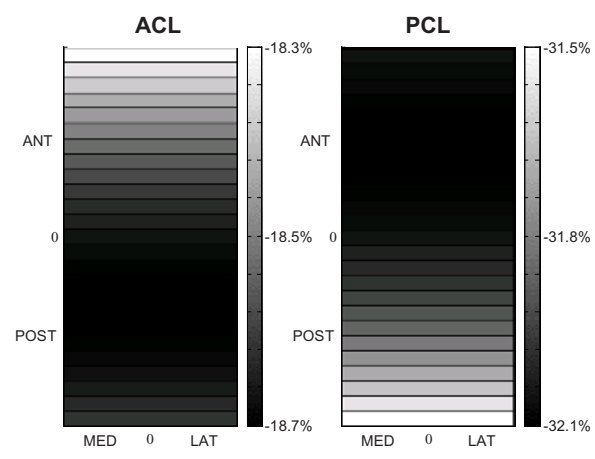

b

Fig. 4. The maximum value of strain over the section of modelled ligament during the execution of the motor task for each of the 25 fibres is plotted for model 1(a) and model 2(b)

The strain calculated for the other fibres resulted also model dependent, in particular the bi-dimensional models produced different results with respect to the three-dimensional ones.

For the bi-dimensional models (Fig.4) the PCL showed the largest strain at the anterior fibres independently from the twist. The strain of the fibres of the ACL was larger for the anterior ones when untwisted and for the posterior ones when twisted.

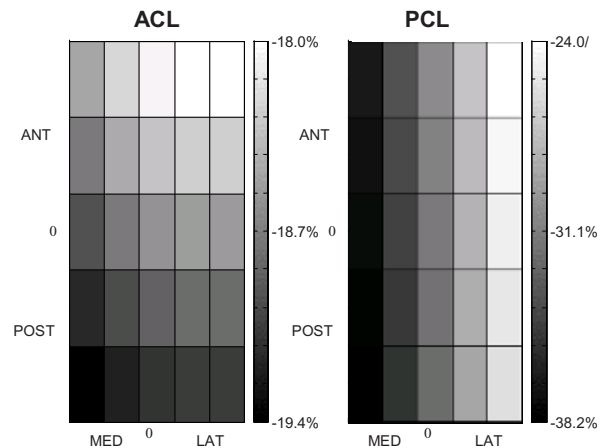

a
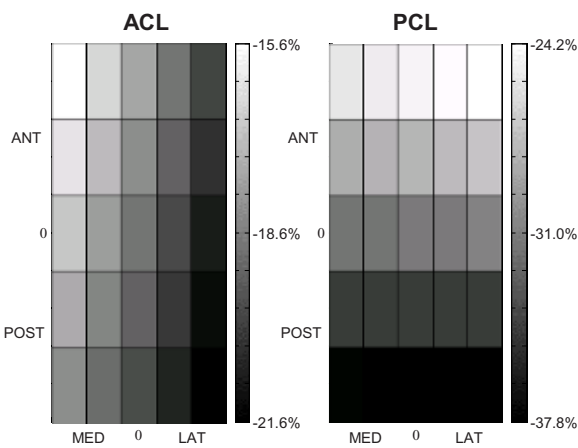

$\mathrm{b}$

Fig. 5. The maximum value of strain over the section of modelled ligament during the execution of the motor task for each of the 25 fibres is plotted for model 3 (a) and model 4 (b)

The strain behaviour of the fibres was similar for the two three-dimensional models (Fig.5 and Fig.6). The largest strain resulted for the ACL at the postero-medial fibres when untwisted, and for the postero-lateral ones when twisted, for the PCL the medial fibres when untwisted and for the posterior fibres when twisted.

\section{Discussion}

Six different cruciate ligament models were compared using parameters from a single selected subject analysed as accurately as possible. Plane, rectangular and circular 
sections were considered, and the mechanical effect of the anatomical twisting of the ligament fibres was also investigated.

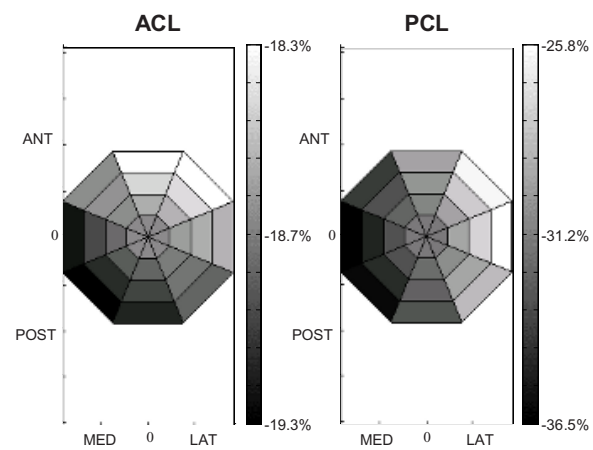

a

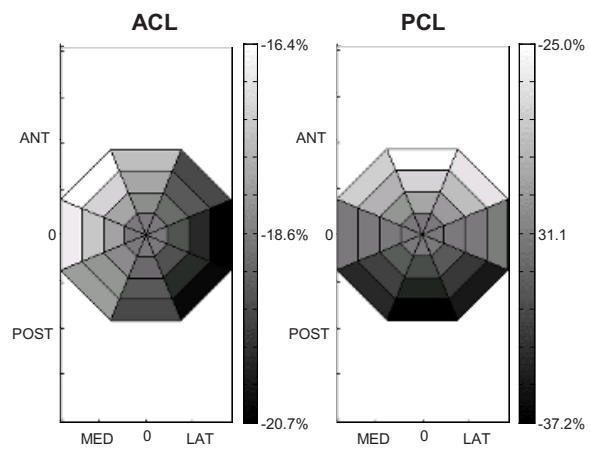

b

Fig. 6. The maximum value of strain over the section of modelled ligament during the execution of the motor task for each of the 25 fibres is plotted for model 5 (a) and model 6 (b)

The strain range of the modelled fibres was not relevantly influenced by the model adopted, which resulted to influence the geometrical distribution of the strain over the fibres in the section of the ligament.

The more conventional bi-dimensional model [10] showed the largest differences from the two three-dimensional ones. No significant difference could be highlighted between the rectangular insertion and the circular insertion three-dimensional models.

The twist showed significant influence in the strain distribution for each model.

In conclusion, when only the magnitude of the fibres elongation is to be calculated the selected model does not considerably affect the results. Instead, the model should be accurately selected when the geometrical distribution of the strain over the section of the ligament is required, i.e. when the strain is used for the calculation of the load applied to the joint by the ligament [11]. In this case, a three-dimensional model is suggested, independently from the selected insertion shape, and the anatomical twist of the fibres has to be taken into account, as it strongly influences the strain distribution over the section.

\section{References}

1. Gill, H.S., O'Connor, J.J.: Biarticulating two-dimensional computer model of the human patellofemoral joint. Clin Biomech 11 (1996) 81-89

2. Lu, T.W., O'Connor, J.J.: Lines of action and moment arms of the major force-bearing structures crossing the human knee joint: comparison between theory and experiment. $\mathrm{J}$ Anat 189 ( Pt 3) (1996) 575-585

3. Huss, R.A., Holstein, H., O'Connor, J.J.: The effect of cartilage deformation on the laxity of the knee joint. Proc Inst.Mech.Eng [H.] 213 (1999) 19-32

4. Kwak, S.D., Blankevoort, L., Ateshian, G.A.: A Mathematical Formulation for 3D QuasiStatic Multibody Models of Diarthrodial Joints. Comput Methods Biomech Biomed Engin. 3 (2000) 41-64 
5. Mommersteeg, T. J., Blankevoort, L., Huiskes, R., Kooloos, J. G., Kauer, J. M.: Characterization of the mechanical behavior of human knee ligaments: a numericalexperimental approach. J Biomech 29 (1996) 151-160

6. Mommersteeg, T.J., Huiskes, R., Blankevoort, L., Kooloos, J.G., Kauer, J.M., Maathuis, P.G.: A global verification study of a quasi-static knee model with multi- bundle ligaments. J Biomech 29 (1996) 1659-1664

7. Piazza, S.J., Delp, S.L.: Three-dimensional dynamic simulation of total knee replacement motion during a step-up task. J Biomech Eng 123 (2001) 599-606

8. Gronenschild, E.: The accuracy and reproducibility of a global method to correct for geometric image distortion in the x-ray imaging chain. Med.Phys. 24 (1997) 1875-1888

9. Zuffi, S., Leardini, A., Catani, F., Fantozzi, S., Cappello, A.: A model-based method for the reconstruction of total knee replacement kinematics. IEEE Trans.Med.Imaging 18 (1999) 981-991

10. Zavatsky, A.B., O'Connor, J.J.: A model of human knee ligaments in the sagittal plane. Part 1: Response to passive flexion. Proc Inst.Mech.Eng [H.] 206 (1992) 125-134

11. Zavatsky, A.B., O'Connor, J.J.: A model of human knee ligaments in the sagittal plane. Part 2: Fibre recruitment under load. Proc Inst.Mech.Eng [H.] 206 (1992) 135-145 